\title{
SELF-ORGANIZING AND SELF-REPAIRING MASS MEMORIES FOR AUTOSOPHY MULTIMEDIA ARCHIVING SYSTEMS Replacing the Data Processing Computer with Self-Learning Machines based on the Autosophy Information Theory
}

\author{
Klaus Holtz, Eric Holtz, Diana Kalienky \\ Autosophy, 602 Mason \#305, San Francisco, California 94108, USA \\ Email:holtzk@autosophy.com, www.autosophy.com
}

Keywords: Autosophy, Information Theory, Multimedia Archiving, Self-Assembling Data Networks, Self-Repairing Memories, Content Addressable Memories, Artificial Intelligence.

\begin{abstract}
The programmed data processing computer may soon be eclipsed by a next generation of brain-like learning machines based on the "Autosophy" information theory. This will require a paradigm shift in memory technology, from random addressable memories to self-organizing failure-proof memories. The computer is essentially a blind calculating machine that cannot find "meaning" as our own brains obviously can. All that can be achieved are mere programmed simulations. The problem can be traced to an outdated (Shannon) information theory, which treats all data as "quantities." A new Autosophy information theory, in contrast, treats all data as "addresses." The original research explains the functioning of self-assembling natural structures, such a chemical crystals or living trees. The same principles can also grow self-assembling data structures that grow like data crystals or data trees in electronic memories without computing or programming. The resulting brain-like systems would require virtually unlimited capacity, failure-proof memories. The memories should be self-checking, self-repairing, self-healing, clonable, both random and content addressable, with low power consumption and very small size for mobile robots. Replacing the programmed data processing "computer" with brain-like "autosopher" promises a true paradigm shift in technology, resulting in system architectures with true "learning" and eventually true Artificial Intelligence.
\end{abstract}

\section{INTRODUCTION}

The programmed data processing computer is now the only known computing paradigm. It is essentially a blind calculating machine that cannot find "meaning" like our brain. All data is regarded as "quantities" according to the Shannon information theory (Shannon, 1948). An alternative computing paradigm is now evolving based on the "Autosophy" information theory (Holtz-Langheld, 1978). All data is regarded as "addresses". The new brain-like machines do not require programming or human supervision of internal functioning. The systems are essentially error-proof, including self-organizing, self-healing, and even self-replicating capabilities. The systems will not produce faulty information even after severe physical damage.

Autosophy theories and applications are described in many scientific publications available on the Internet. (Use keyword "autosophy" in a search engine or go to www.autosophy.com.)
A self-learning text database was built in 1988 to verify the theoretical predictions. Autosophy Internet television was demonstrated in 2000 (Holtz, 2001). Primitive applications include lossless data compression in the V.42bis modem standard and lossless still image compression in the gif and tif standards.

The new Autosophy technology would require a new memory paradigm, which is the main subject of this paper. Random Addressable Memories, used in computers, are not suitable. The new mass memory systems would provide virtually unlimited, cheap and non-volatile storage capacities, combined with very low power consumption and small size for use in mobile robots (Holtz, 2003).

Both the theoretical knowledge and the required memories are now becoming available for building brain-like systems (Holtz, 2004). This may evolve into self-learning robots and eventually into true Artificial Intelligence. It may also revolutionize all multimedia communication (Holtz, 2002). 


\section{COMPUTER VS. AUTOSOPHER}

The programmed data processing computer in Fig. 1 is essentially a blind adding or calculating machine that cannot find "meaning" as our own brains obviously can. All input and output data items (ASCII characters or pixels) are regarded as quantities according to the Shannon information theory. Its purpose is to combine raw input data, using arithmetic, according to a stored program, to obtain useful output data. The computer cannot learn and no matter how much data is processed or stored it will not become more intelligent. All intelligence is contained in the programming, prepared by human programmers, where every operation must be defined by a complex series of instructions. No matter the speed of the computation or the complexity of the software, Artificial Intelligence in a computer will always remain a mere simulation.

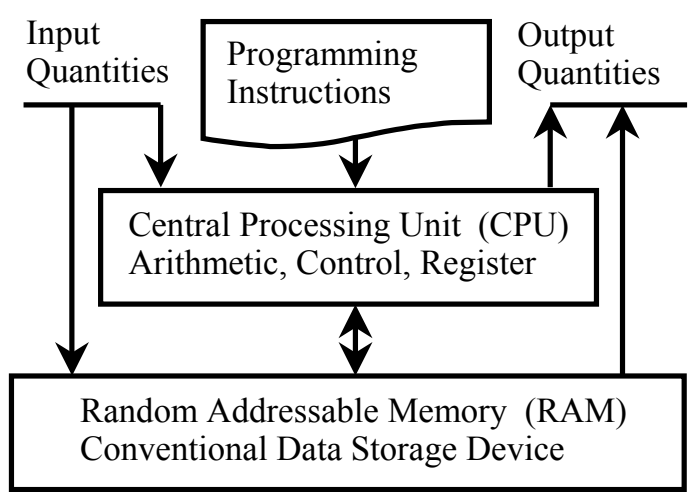

Figure 1: Programmed data processing computer

Computers store data in a Random Addressable Memory (RAM). The address and data content are both provided by the Central Processing Unit (CPU), according to the programmed instructions. Data may enter or exit the computer either via the CPU or directly via Direct Memory Access (DMA). Both the program data and the input/output data may be stored in the same memory device. This may cause viruses from the communication channels to infest the computer. A single error in a computer memory may lead to a total computer malfunction.

The Autosophy theory evolved from research into self-assembling structures, such as chemical crystals, living trees, or human societies. These structures may start from a tiny seed containing information stored in a DNA helix. The seed will select specific materials from a random environment to build itself into a very complex structure without human design or supervision. In 1974 Klaus Holtz discovered that the same natural laws and principles grow selfassembling data structures in memories.
These imaginary data structures grow like data crystals or data trees in electronic memories, without programming or outside supervision. The Autosophy information theory was first disclosed in 1978 (Holtz-Langheld, 1978).

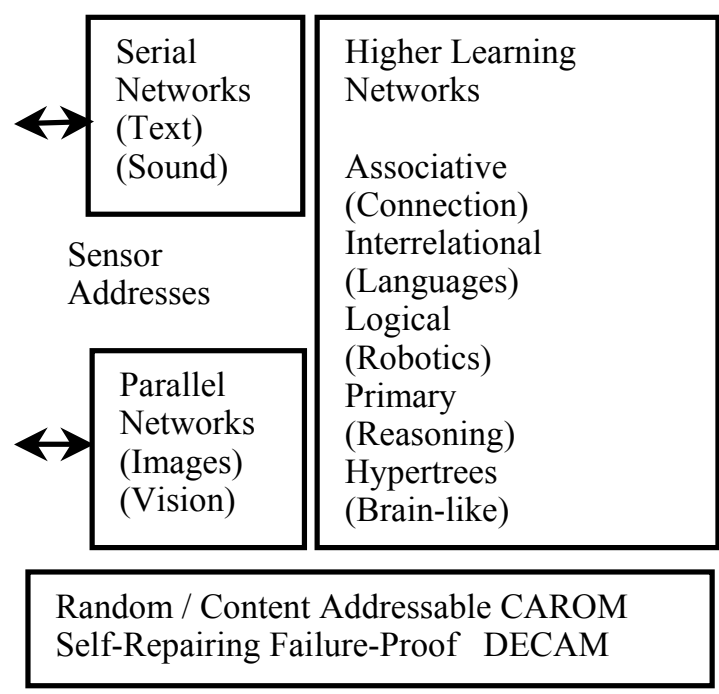

Figure 2: Self-learning brain-like Autosopher

The operations in an "autosopher", shown in Fig. 2, are based on self-learning networks. The device acts like a "black box" to absorb all multimedia information without programming. The sensor input/output data are regarded as "addresses," which define or create their own storage locations in the memory. Computer programming is replaced by "education" similar to the education of children. Simple input networks, such as the serial and the parallel networks, encode the raw sensor data for further processing in higher level learning networks. All learning networks may share the same mass memory device. The memory must be virtually error-proof for applications in self-learning intelligent robots.

\section{SELF-LEARNING NETWORKS}

The serial network, shown in Figure 3, is an example of true mathematical "learning," according to the Autosophy information theory. This can be imagined like the growing of data trees or data crystals where data input items define or create their own storage locations in the memory. A unit of knowledge (engram) is created by new information (GATE), related to already established knowledge (POINTER), which may then create a new engram (ADDRESS) as an extension to that which is already known (Patent 4,366,551). 


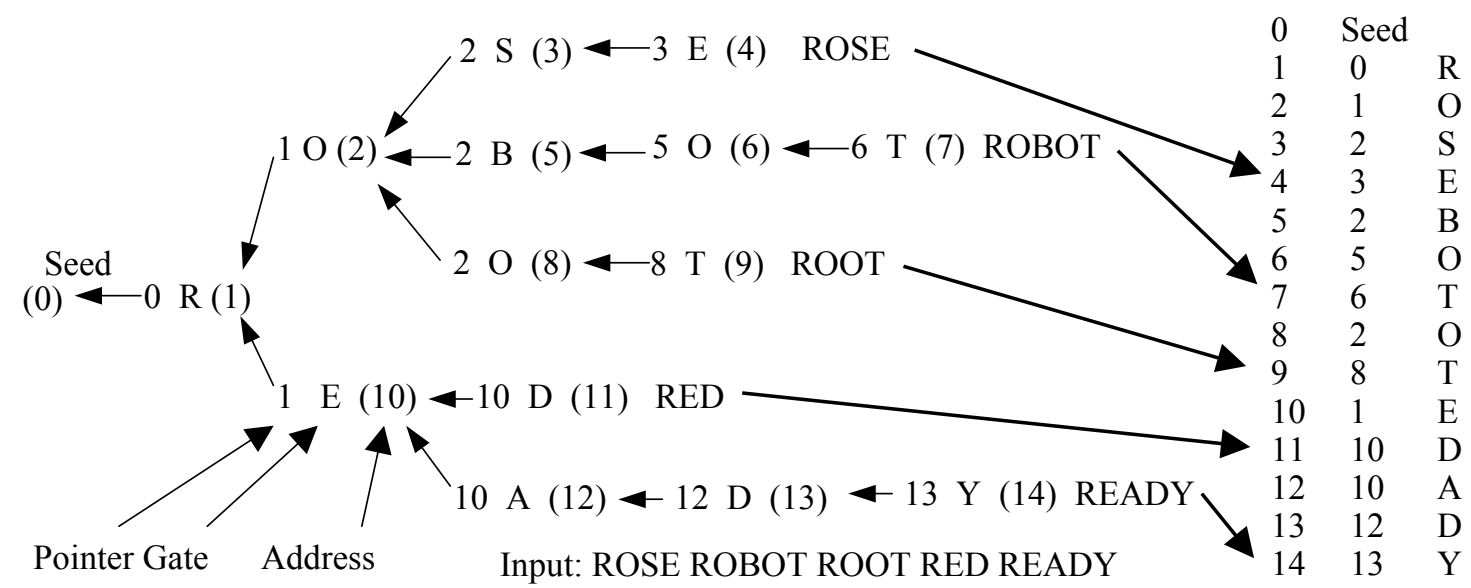

\section{SERIAL NETWORK LEARNING ALGORITHM \\ MATRIX [ POINTER ] GATE ] (The MATRIX is a working register in the hardware)}

Start: Set POINTER $=$ Seed $(=0)$

Loop: Move the next input character into the GATE

If End Of Sequence (a SPACE char.) then output the POINTER as a Tip code; Goto Start

Else search the library for a matching MATRIX

If found then move the library ADDRESS where it was found to the POINTER; Goto Loop

Else, if not found, then store the MATRIX into a next empty library ADDRESS;

Move the library ADDRESS where it was stored into the POINTER: Goto Loop

SERIAL NETWORK RETRIEVAL ALGORITHM

MATRIX [ POINTER ] GATE ]

Start: Move the input Tip code into the POINTER

Loop: Use the POINTER as a library ADDRESS to fetch a next MATRIX from the library

Push the GATE into a First-In-Last-Out (FILO) stack

If the POINTER $=$ Seed $(=0)$ then pull the output data from the FILO stack; Goto Start Else Goto Loop

Figure 3. An example of the "Serial" self-learning tree network (Patent 4,366,551)

The learning process can be imagined like the growing of data trees or data crystals. A stored tree network is made of separate nodes, where each ADDRESS represents an "engram" of knowledge. The library ADDRESS is a mathematical equivalent to a point in omni dimensional hyperspace. The content of each library ADDRESS is unique and can be stored only once. One cannot learn what one already knows. Because each input data string is learned only once, the storage requirements would saturate. The more information already contained in a database the less additional storage space is required to store additional information.

There are seven known classes of self-learning "Omni Dimensional Networks", each providing a different learning mode. This may include learning modes that are not available in our own brains. Some of these learning networks are already implemented in commercial applications, while others have been simulated or are known only in theory. New applications, such as live Internet video or advanced lossless still image compression, are now being added at an accelerating rate. $\underline{\text { Serial networks }}$ store serial data sequences such as text, sound, or serially scanned images. The algorithm was invented in 1974 (Patent 4,366,551). A similar algorithm (LZ-78) was developed later by Jacob Ziv and Abraham Lempel (Ziv-Lempel, 1978). Most commercial applications use the LZW (Lempel Ziv Welch) code, a simplified variation published by Terry Welch (Welch, 1984). Application examples include the V.42bis data compression standard in modems and the gif and tif formats used for lossless still image compression.

Parallel networks store images in a hyperspace funnel, yielding very high lossless image compression and fast access to archives. These networks are especially suitable for archiving and storage of imaging and video data. Machine vision is the ultimate application.

Associative networks connect various networks into a system. They can, for example, connect questions to answers, text to images, or commands to execution sequences. A demonstration system was built in 1978 to verify operations. 
Interrelational networks provide grammatical language learning that could evolve into talking databases with speech input and output. Grammatical speech would be the ultimate method of communication between humans and machines.

Logical networks yield an advanced form of selflearning data processing with logical reasoning capabilities. They may evolve into intelligent robots.

Primary networks provide unstructured access to archives or databases through deductive reasoning and automatic indexing.

Hypertree networks promise true brain-like learning, which is currently being researched. This ongoing research may add new types of networks and new learning modes in the future.

The learning algorithms require that the entire memory must be searched to locate a matching MATRIX before creating a new network node. Rapid learning would require a Content Addressable Memory (CAM) in which all memory locations are searched in parallel, all in one operation. The retrieval algorithm, in contrast, would require a normal Random Addressable Memory (RAM). The optimal solution is to store each node in two separate memory devices, one configured as a CAM, the other as a RAM. This would also provide for automatic self-repair in failure-proof memories.

When Autosopher robots finally evolve from mere information access terminals to physical interactions with human beings, then near-absolute reliability is essential even in cases of severe physical damage. A malfunctioning robot may cause severe damage and injury to human beings. Robots would require enormous capacity, non-volatile, Content or Random Addressable memories. The memory units should be small enough to fit into mobile robots and consume very little power so as to require no cooling and conserve the limited power of mobile terminals.

The memory units should have very rugged construction for operation in hostile environments and even in outer space. Maintenance could be by unskilled personnel and eventually by remote service robots. Only solid-state technology is used, without any moving parts. The outer surface may be sculptured for better heat transfer and easier handling by human hands or robots during memory repair. The input output bus connector may be similar to the Edison socket now used for light bulbs. Memory modules may be screwed-in or removed, like light bulbs, without any additional mounting hardware. There are two types of memory units, a "female" unit (CAM), and a "male" unit (RAM), with the same bus sockets, either male or female, on both sides. The memory units could be stacked into larger and larger groups to provide virtually unlimited storage capacity.

\section{CONTENT ADDRESSABLE READ ONLY MEMORY}

The CAROM memory, shown in Fig. 4, may operate as a Content Addressable Memory (CAM) or as a Random Addressable Memory (RAM). Both the memory address and the stored data are programmable from the outside by tiny fuses. The fuses are made either conductive or nonconductive according to the Autosophy learning algorithms, where the data determines or creates its own storage nodes. There is no need for programming or outside supervision of the internal operations.

Memory addresses are decoded in long chains of Field Effect Transistors (FETs), where each transistor is turned conductive or nonconductive as selected by a pair of fuses. Only one fuse in the pair is made conductive. A complementary input voltage is applied through the conductive fuse to the FETs gate for either conductive or nonconductive. If all FETs in a chain are conductive, then current will flow through the FETs to a set of diodes to generate the output data via programmable output fuses. Only one chain of FETs can conduct at any one time according to the learning algorithms. An input address will therefore produce an output data pattern as determined by the learning algorithms.

Since only one chain of FETs can conduct at any one time, the current through the memory device is constant and independent of the memory capacity. The voltage input from the driver charges the tiny capacitance on the FETs gate. The input acts like a pure capacitive load where the whole memory device acts like a large capacitor.

The energy used to charge the capacitor can be recycled through an inductor to achieve very low energy consumption. When the stored capacitive voltage is discharged through the driver, in a charge reversal, then the energy is temporarily stored in an inductor. The energy stored in the inductor then recharges the capacitive load in the opposite polarity. This is similar to an electrical pendulum, where the energy is being recycled in each charge reversal. The result is very low net energy loss only in the conductive layers and in the FETs.

Very small net energy consumption will prevent heating and conserve the limited power in mobile robots. Like the human brain, power consumption will increase with memory activity, such as learning and thinking. However, power consumption is virtually independent of the memory capacity. A very large memory device may have virtually the same power consumption as a small memory device. The internally stored energy in the capacitor may even allow a robot to continue operations after short power failures. 


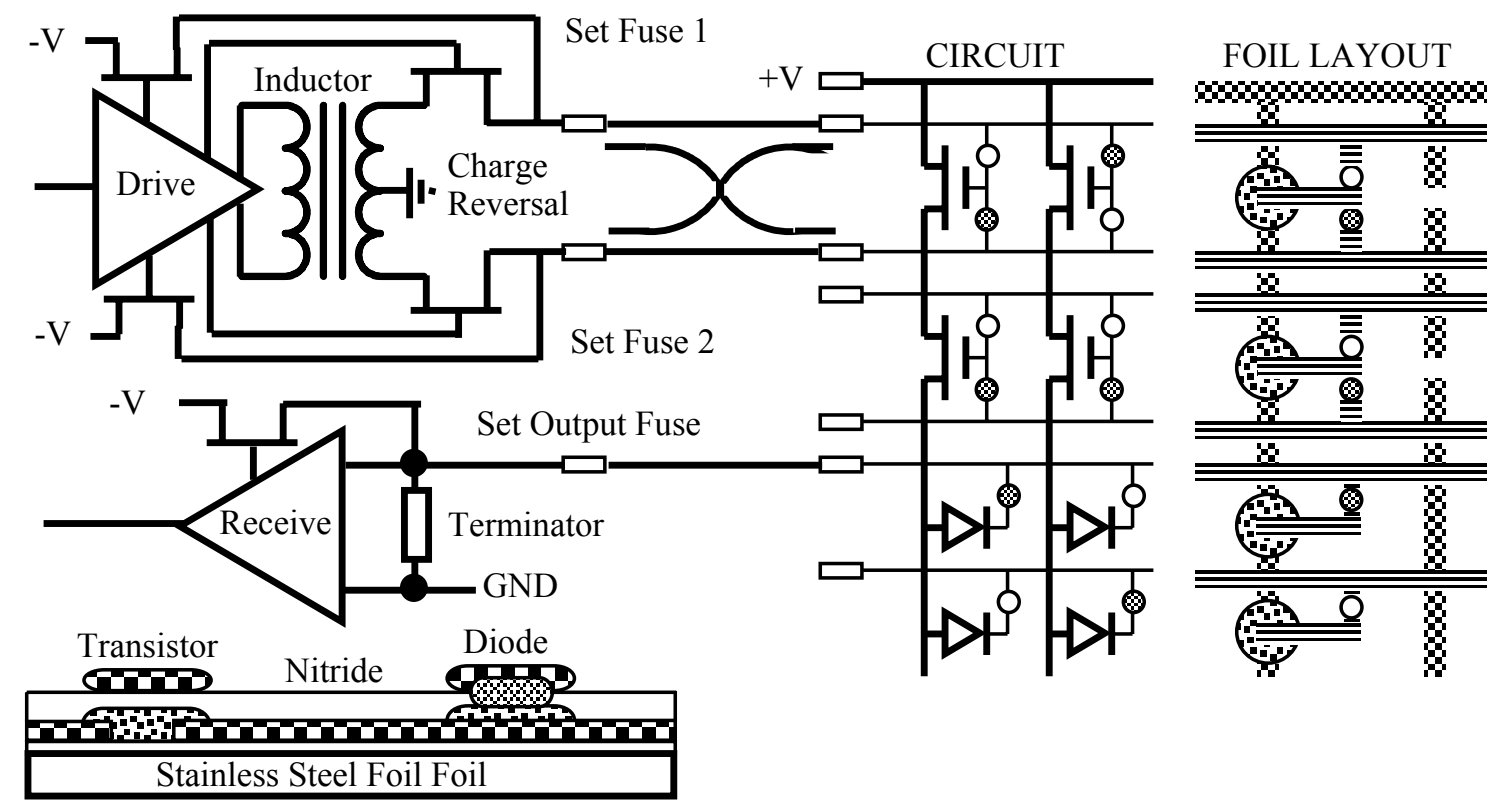

Figure 4: CAROM Memory using Thin Film FET Semiconductor and Fuses (Patent 5,576,985)

The memory circuits are printed onto thin foils, which are rolled into a spool, about the size of a roll of toilet paper. The spools should only contain the printed foil as shown in the FOIL LAYOUT without active components. The driver and receiver circuits should be housed in a separate bus connector unit in the center of the spool. Rolling the foil into a tight spool would provide very rugged and compact construction. The spool is then inserted into a capsule filled with self-sealing liquid.

The memory fuses are set by the learning algorithm whenever a new node is created and stored in a "next empty" memory location. Each node may be stored anywhere in the memory device. Once a fuse is set, from the driver or receiver, then it will usually remain set to form a read-only memory. All address bits and the output data bit fuses in a network node may be set in a single cycle. Fuse programming will use larger voltages and currents than normal memory searching operations. Each column of fuses in a node may be specified in a "next empty" word selection layer on the foil.

There are several available fuse technologies: conventional non-volatile memories (PROM, EPROM, EEPROM, FLUSH) may change over long time periods and are not recommended. Antifuses are used in Field Programmable Gate Arrays (FPGAs), which are more stable and less sensitive to heat or radiation. Fuses made from electro-active PLZT (Lead, Lanthanum, Zinc, Titanium) ceramics may be best for long-term memories. Some fuse technologies may allow for memory erasure and recycling of the spools.
There are three basic technologies for manufacturing the memory spools: Silicon chips mounted on a laminated foil would provide the cheapest solution at this time. The circuit shown in Fig. 4 would be printed on crystalline silicon chips for separate mounting on the foil. This may yield a price of about $\$ 1$ per billion transistors. (The Intel Pentium 4 contains 330 billion transistors and sells for $\$ 350$ ). The silicon chips need not be error-free because of the DECAM's self-repair capabilities.

Thin film amorphous silicon transistors and interconnecting wiring may be printed by vacuum deposition onto stainless steel foil. This roll-to-roll industrial process may result in very inexpensive memory units that can withstand great heat, radiation, and vibrations.

Printable electronics circuits are printed on plastic foils by inkjet printing processes. Special semiconductive ink can produce the wiring and organic transistors, diodes, and PLZT fuses.

\section{DUAL ENTRY MEMORIES}

Both the autosopher and the brain store multimedia "information" in a saturating omni dimensional hyperspace format, in which any node may be located anywhere. Memory repair is far beyond human intelligence. Repairing an individual hyperspace memory node is just as impossible as repairing individual neurons in the brain. Both systems act like a sealed "black box" to organize and repair their own memory operations. 
Examples of dual redundant information storage are found in double ledger accounting and the DNA helix. In double ledger accounting every transaction is recorded twice, as a gain and as a loss. Errors in one ledger can be corrected from the other ledger to obtain error proof accounting. In biological DNA, information is stored in two strands wound together into a helix, where each strand contains the same information but in a complementary form.

Autosopher store information in two spools, a male (RAM) and a female (CAM), each containing the same information in a complementary format. An error in one spool is automatically repaired from the complementary spool. Memory repair clears defective nodes, which are repaired in sleep mode.

Each search or retrieval memory access involves both spools. In a search access, a MATRIX is applied to the CAM, resulting in an ADDRESS where a match was found. The ADDRESS is applied to the RAM, resulting in a MATRIX, which must be identical to the original MATRIX input. In retrieval, an ADDRESS is applied to the RAM, resulting in a MATRIX output, which is applied to the CAM to result in an ADDRESS, which must be identical to the original ADDRESS input. An error in either spool, confirmed by a "checksum" (A Hamming code of both the MATRIX and the ADDRESS), would clear the node for later repair. Defective memory nodes cleared during normal operations are repaired in "sleep" mode.

Self-healing involves applying a binary counter to the CAM to retrieve an ADDRESS and checksum from the CAM. If the checksum code is incorrect or empty, then the counter is incremented to check the next node. Otherwise the output ADDRESS is applied to the RAM to obtain a MATRIX output, which must be identical to the counter ADDRESS. Else the node in the CAM is used to generate a new node in the RAM in a "next empty" memory location. Once the CAM has been scanned then the binary counter ADDRESS is applied to the RAM ADDRESS. This will result in a MATRIX output, which is applied to the CAM. The CAM ADDRESS output must be identical to the counter ADDRESS.

The automatic self-healing facilities can also be used for rejuvenation and cloning of robot memories. Removing one spool and replacing it with an empty spool will cause a robot to automatically restore the information from the remaining spool into the empty spool. The removed spool may then be inserted into a second robot, together with an empty spool, to produce a robot clone with the same knowledge and "personality." Rejuvenation involves double cloning by removing an old spool and replacing it with an empty spool. Old robots can thus be rejuvenated without loss of information.

\section{CONCLUSIONS}

Considering the enormous head start of the programmed data processing computer, replacing the computer with self-learning autosopher will neither be quick nor easy. However, there may not really be a choice. Computer technology is approaching the limits of performance where further progress will require more and more effort. Higher and higher speed computation, more advanced operating systems, and better programming are not the solution. Shannon's "communication," using binary digits, is not true communication, and the programmed data processing computer will not achieve true Artificial Intelligence. It may be hard to accept that our entire communications infrastructure and the programmed data processing computer are based on a false (Shannon) information theory.

The Autosophy information theory, in contrast, is based on self-learning networks that grow like data crystals or data trees in electronic memories. The intelligence of archiving systems or robots is no longer limited by the intelligence of the human programmers. Autosophy may provide an alternate computing and communication paradigm for the future, from human-designed and programmed machines, towards self-organizing and self-repairing brain-like machines.

\section{REFERENCES}

Holtz, K., 2004. Autosophy Failure-Proof Multimedia Archiving. IS\&T's 2004 Archiving Conference. www.imaging.org, San Antonio Texas, April, (2004)

Holtz, K., 2003. Replacing Data Processing Computer with Self-Learning Autosopher: Impact on Communication and Computing. SCI 2003 The 7th World Multi-conference on Systemics, Cybernetics and Informatics. Orlando FL. July (2003)

Holtz, K., 2002. The Emerging Autosophy Internet. SSGRR 2002s, L'Aquila Italy Aug. 2 (2002) www.ssgrr.it/en/ssgrr2002s/papers.html, Paper 140.

Holtz, K. 2001. An Autosophy Image Content-Based Television System. IS\&T's 2001 PICS Montreal (2001)

Welch, T., 1984. A Technique for High Performance Data Compression. IEEE Computer, June (1984)

Ziv, J., Lempel A., 1978. Compression of Individual Sequences via Variable-Rate Coding. IEEE Information Theory, IT-24 (1978)

Holtz, K., Langheld, E., 1978. Der selbstlernende und programmier-freie Assoziationscomputer. ELEKTRONIK magazin, Germany, Dec. (1978).

Shannon, C., 1948. A mathematical Theory of Communication. Bell Telephone B-1598, July (1948) 